\title{
Hallazgos coronarios y no coronarios en Angio-TC de arterias coronarias: Revisión de 87 casos
}

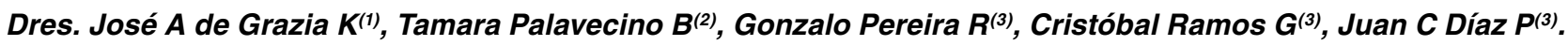

1. Becado de Radiología, Centro de Imagenología. Hospital Clínico Universidad de Chile. Santiago, Chile.

2. Fellow, Unidad de Tórax, Centro de Imagenología. Hospital Clínico Universidad de Chile. Santiago, Chile.

3. Staff, Unidad de Tórax, Centro de Imagenología. Hospital Clínico Universidad de Chile. Santiago, Chile.

\section{Classification of Coronary Artery Disease Using CT-Coronary Angiogram: Review of 87 Cases}

\begin{abstract}
Objective. To describe and classify coronary and non-coronary cardiovascular findings using CT Angiography of coronary arteries in a group of patients treated at the University of Chile Clinical Hospital. Materials and Methods: We retrospectively reviewed 87 CT Angiograms of coronary arteries. The presence of coronary atheromatous disease and its severity was recorded and classified as non significant $(<50 \%)$ or significant stenosis (>50\%), subdividing this latter group into moderate (51-70\%), severe (71-99\%) or occlusion (100\%). In each patient the number of affected arteries was recorded. The presence of coronary stents and bypass was analyzed, showing their location and permeability. Non-coronary cardiovascular findings were recorded. Results. A total of 51 patients had coronary atheromatous disease that was able to be classified using CT Angiography. Of these, 14 (27\%) showed non significant stenosis and 37 (73\%) significant stenosis. In the cases with significant stenosis 19 were moderate, 11 severe and 7 occlusion. The number of affected arteries was one in $43 \%$ of the patients, two in $39 \%$ and three in $18 \%$. Five patients had coronary stents, one of those occluded. Three patients had triple coronary bypass, each with at least one occluded graft. Fourteen patients showed non-coronary cardiovascular findings, the most common being aortic valve disease. Conclusion. The CT Angiogram of coronary arteries is a useful examination to classify atheromatous coronary disease and determine its severity. It is useful for evaluating coronary stents and bypass, and to determine their permeability. It also provides information relating to non-coronary diagnosis on cardiac, aortic and pericardial levels.
\end{abstract}

Keywords: Computed tomography, Coronary atherosclerosis.

Resumen: Objetivo. Describir y caracterizar los hallazgos coronarios y cardiovasculares no coronarios en Angio-TC de arterias coronarias de una cohorte de pacientes atendidos en el Hospital Clínico de la Universidad de Chile. Materiales y método. Se revisaron retrospectivamente 87 Angio-TC de arterias coronarias. Se registró la presencia de enfermedad ateromatosa coronaria y su severidad, clasificándola en estenosis no significativa (<50\%) o significativa (>50\%), subdividiendo a este último grupo en moderada (51-70\%), severa (71-99\%) u oclusión (100\%). En cada paciente se registró el número de arterias comprometidas. Se analizó la presencia de stents y bypass coronarios, señalando su localización y permeabilidad. Se registraron hallazgos cardiovasculares no coronarios. Resultados. Un total de 51 pacientes presentó enfermedad ateromatosa coronaria caracterizable por Angio-TC. De éstos, 14 (27\%) mostraron estenosis no significativa y $37(73 \%)$ estenosis significativa. En los casos con estenosis significativa 19 fueron moderada, 11 severa y 7 oclusión. El número de arterias comprometidas fue una en el $43 \%$ de los pacientes, dos en el $39 \%$ y tres en el 18\%. Cinco pacientes presentaban stents coronarios, uno de ellos ocluido. Tres pacientes presentaban triple bypass coronario, cada uno con al menos un puente ocluido. Catorce pacientes mostraron hallazgos cardiovasculares no coronarios, siendo el más frecuente la valvulopatía aórtica. Conclusión. El Angio-TC de arterias coronarias es un examen útil para caracterizar la enfermedad ateromatosa coronaria y determinar su severidad. Es útil para evaluar stents y bypass coronarios y determinar su permeabilidad. Brinda también información sobre diagnósticos no coronarios a nivel cardiaco, aórtico y pericárdico.

Palabras clave: Aterosclerosis coronaria, Tomografía computada. 
de Grazia JA, Palavecino T, Pereira G, Ramos C, Díaz JC. Hallazgos coronarios y no coronarios en Angio-TC de arterias coronarias: Revisión de 87 casos. Rev Chil Radiol 2014; 20(3): 96-104.

Contacto: Dr. José A de Grazia K. / ja.degrazia@gmail.com

Trabajo recibido el 06 de abril de 2014. Aceptado para publicación el 09 de junio de 2014.

\section{Introducción}

La cardiopatía coronaria es la principal causa de muerte en Chile ${ }^{(1)}$ y en el resto de los países occidentales $^{(2)}$. La coronariografía constituye el gold standard para el estudio de la enfermedad ateromatosa de las arterias coronarias, sin embargo, es un examen invasivo, no exento de complicaciones ${ }^{(3)}$. Con el advenimiento de la TC multidetector, en especial con equipos de 64 o más canales, ha sido posible estudiar la anatomía del corazón y las arterias coronarias como nunca antes, de forma no invasiva, rápida y con un alto rendimiento diagnóstico ${ }^{(4-8)}$. La sensibilidad y especificidad del método para el diagnóstico de enfermedad ateromatosa coronaria hemodinámicamente significativa se elevan por sobre el $95 \%$ y el valor predictivo negativo puede alcanzar el $99-100 \%{ }^{(9,10)}$. Además de esto, el Angio-TC de arterias coronarias es hoy en día el examen de elección para el estudio de variantes anatómicas del origen de las arterias coronarias ${ }^{(11)}$, algunas de las cuales pueden tener serias repercusiones hemodinámicas sobre la circulación coronaria. También se ha descrito su utilidad en la evaluación de la permeabilidad de stents y bypass coronarios ${ }^{(7)}$.

En Chile se ha realizado un solo estudio descriptivo enfocado a hallazgos cardíacos no coronarios en Angio-TC de arterias coronarias ${ }^{(12)}$. No existen trabajos que describan los hallazgos de la enfermedad ateromatosa coronaria, variantes anatómicas del origen de las arterias coronarias, ni hallazgos en presencia de stents y bypass coronarios, situaciones en las cuales el estudio ha demostrado ser de utilidad. Resultan necesarios estudios nacionales que ayuden a comprender las potencialidades diagnósticas de la técnica, sus limitaciones y los escenarios en que puede presentar mayor utilidad clínica.

El objetivo del presente estudio es describir y caracterizar los hallazgos coronarios y cardiovasculares no coronarios en Angio-TC de arterias coronarias de una cohorte de pacientes atendidos en el Hospital Clínico de la Universidad de Chile.

\section{Materiales y método}

Se revisaron en forma retrospectiva 87 Angio-TC de arterias coronarias realizados en el Hospital Clínico de la Universidad de Chile entre los años 2009 y 2012, con un tomógrafo computado SOMATOM Sensation ${ }^{\circledR}$ (Siemens ${ }^{\circledR}$ ) de 64 canales. Todos los exámenes fueron realizados con frecuencia cardiaca menor o igual a $65 \mathrm{lat} / \mathrm{min}$, obtenida en los casos necesarios mediante ß-bloqueo con Atenolol VO (50$100 \mathrm{mg}$ 1-2 horas previo al examen). Se administró también Nitroglicerina $0,6 \mathrm{mg} \mathrm{SL}$ o Isosorbide 10 $\mathrm{mg} \mathrm{VO}$ al momento del examen, como vasodilatador coronario. El protocolo de examen incluyó una fase no contrastada con adquisición secuencial y gatillado electrocardiográfico prospectivo para cálculo del índice de calcio (score de Agatston), además de una fase angiográfica con adquisición volumétrica y gatillado electrocardiográfico retrospectivo. El field of view (FOV) se extendió desde la carina hasta el borde inferior del corazón; en el estudio de bypass coronarios el límite superior del FOV se extendió cranialmente hasta las clavículas. Para la fase angiográfica se utilizó medio de contraste yodado no-iónico, en dosis de $1 \mathrm{ml} /$ $\mathrm{kg}$, previo a la realización de un bolus test en la raíz de la aorta. La inyección de medio de contraste se realizó a través de una vía venosa periférica de 18 $G$ en la fosa antecubital derecha, a una velocidad de entre 5 y $7 \mathrm{ml} / \mathrm{seg}$, realizada en forma bifásica, es decir, seguida de un bolo de solución fisiológica. Los parámetros técnicos del equipo para la realización de la fase angiográfica fueron los siguientes: 120 $\mathrm{Kv}, 900 \mathrm{mAs}$, velocidad de rotación del tubo de 330 mseg, colimación del detector $64 \times 0,6 \mathrm{~mm}$, intervalo de reconstrucción 0,4 mm, pitch 0,2. Las imágenes fueron presentadas en ventana mediastínica, con filtro Kernel medio-fino (B30f o B46f en pacientes con stents). Para la reconstrucción se ocuparon imágenes de meso o telediástole $(60-70 \%$ del intervalo $R-R$ ). En la estación de trabajo las imágenes fueron procesadas, obteniéndose reconstrucciones multiplanares (MPR) y curvilíneas (CPR), además de imágenes con proyección de máxima intensidad (MIP) y reconstrucciones volumétricas (VRT).

Cada uno de los exámenes fue evaluado por un radiólogo de la Unidad de Tórax, con experiencia en Cardio-TC. Se registró la presencia de enfermedad ateromatosa coronaria y su severidad, clasificándola en estenosis no significativa $(<50 \%)$ o significativa $(>50 \%)$, subdividiendo a este último grupo en estenosis moderada (51-70\%), severa $(71-99 \%)$ u oclusión (100\%). En cada paciente se registró el número de arterias comprometidas, considerándose la estenosis más severa para la categorización. Se analizó además la presencia de stents y bypass coronarios, señalando su localización y permeabilidad. Se registraron variantes anatómicas del origen de las arterias coronarias y otras alteraciones coronarias poco frecuentes. Por último, se registró la presencia de hallazgos cardiovasculares no coronarios, incluyendo secuelas de infarto miocárdico, cardiopatías congénitas, valvulopatías, miocardiopatías, patologías de la aorta torácica y alteraciones del pericardio. 


\section{Resultados}

La edad promedio de los pacientes fue 56 años (DE +/- 14). La muestra incluyó 60 hombres (69\%) y 27 mujeres (31\%). El motivo de solicitud del examen más frecuente fue sospecha de enfermedad ateromatosa coronaria (54\%), seguido de enfermedad ateromatosa coronaria conocida (17\%). Las características demográficas de la muestra y los motivos de solicitud del examen se resumen en la tabla $I$.

Respecto a la anatomía coronaria, la dominancia de la circulación coronaria fue derecha en el $87 \%$ de los casos, izquierda en el $7 \%$, codominancia en el $5 \%$ y no clasificable en una paciente con un ventrículo único operado con severa distorsión de la anatomía coronaria. El 5\% de los pacientes presentaron puentes intramiocárdicos. Cinco pacientes mostraron variantes anatómicas del origen de las arterias coronarias (Figura 1), información resumida en la tabla II.

El índice de calcio promedio de la muestra fue de 184 unidades de Agatston (UA). Dos pacientes presentaron índice de calcio mayor a 1000 UA, excluyéndolos del estudio angiográfico. Un total de 51 pacientes presentó enfermedad ateromatosa coronaria caracterizable por Angio-TC. De estos, 14 (27\%) mostraron estenosis no significativa y $37(73 \%)$ estenosis significativa. En los casos con estenosis significativa, 19 (37\%) fueron moderada, 11 (22\%) severa y 7 (14\%) oclusión (Figura 2). El número de arterias comprometidas fue una en el $43 \%$, dos en el $39 \%$ y tres en el $18 \%$ de los pacientes. La arteria más comprometida fue la descendente anterior $(45 \%)$, seguida en orden decreciente por la circunfleja (29\%), la coronaria derecha (20\%) y finalmente el tronco coronario izquierdo (6\%). La información respecto a la enfermedad ateromatosa coronaria se resume en la tabla III.

Cinco pacientes presentaban stents coronarios. Uno de ellos 3 stents, otro 2 stents y los restantes tres un stent (Figura 3). De los 8 stents evaluados, sólo uno se encontraba ocluido. La información respecto a los stents coronarios se resume en la tabla IV.

Tres pacientes presentaban triple bypass coronario.
Tabla I. Características demográficas y motivo de solicitud del examen.

Edad (años)

Promedio (DE)

Edad mínima / máxima $56(+/-14)$ $14 / 86$

Sexo

Hombre 60

Mujer 27

Motivo de solicitud del examen *

Sospecha de enfermedad $54 \%$

ateromatosa coronaria

Enfermedad ateromatosa

coronaria conocida

$17 \%$

Sospecha de anomalía

congénita coronaria

Miocardiopatías

$5 \%$

Cardiopatías congénitas

$3 \%$

Otros

$16 \%$

* Por tratarse de un estudio retrospectivo, la fuente de información del motivo de solicitud del examen fue la orden médica escaneada en el sistema electrónico del Centro de Imagenología. Esta orden médica no estaba disponible o bien no contenía información en 29 pacientes, por lo que el análisis de esta variable fue hecho sólo con 58 pacientes.

Todos ellos tenían un puente de arteria mamaria interna izquierda sobre arteria descendente anterior y dos puentes venosos aorto-coronarios (Figura 4). De los 9 puentes evaluados, 4 se encontraban ocluidos (2 puentes arteriales y 2 puentes venosos). La información respecto bypass coronarios se resume en la tabla $\mathrm{V}$.

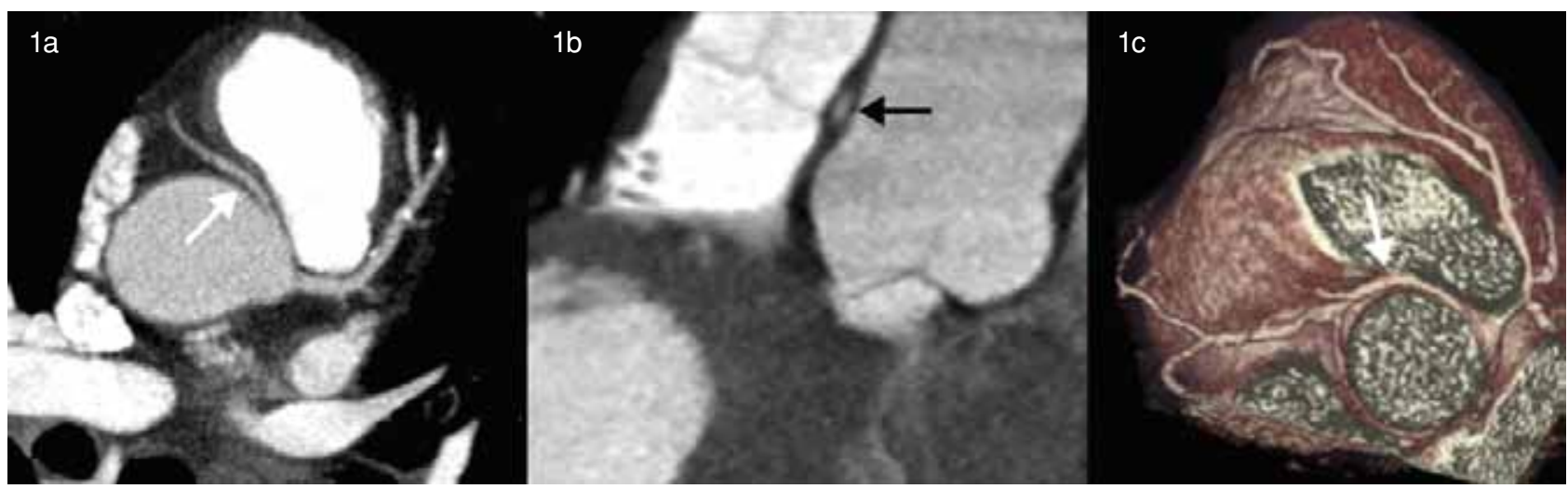

Figura 1. Arteria coronaria derecha originada en el seno de Valsalva izquierdo, de curso interarterial (flecha). a) Corte axial. b) Reconstrucción sagital oblicua. c) Imagen VRT. 
Tabla II. Variantes anatómicas del origen de las arterias coronarias.

\begin{tabular}{|c|c|}
\hline Paciente & Variante anatómica \\
\hline 1 & $\begin{array}{l}\text { ACD originada en la ADA, de curso } \\
\text { prepulmonar }\end{array}$ \\
\hline 2 & $\begin{array}{l}\text { ACD originada en el seno de Valsalva } \\
\text { izquierdo, de curso interarterial }\end{array}$ \\
\hline 3 & $\mathrm{ACl}$ originada en la arteria pulmonar \\
\hline 4 & $\begin{array}{l}\text { ACD hipoplásica, originada en la } \\
\text { aorta ascendente }\end{array}$ \\
\hline 5 & $\begin{array}{l}\text { ACX originada en la ACD, de curso } \\
\text { retroaórtico }\end{array}$ \\
\hline \multicolumn{2}{|c|}{$\begin{array}{l}\text { ACD: arteria coronaria derecha, } \mathrm{ACl} \text { : arteria coronaria izquierda } \\
\text { ADA: arteria descendente anterior, } \mathrm{ACX} \text { : arteria circunfleja. }\end{array}$} \\
\hline
\end{tabular}

Tabla IV. Stents coronarios.

\begin{tabular}{|c|c|c|l|}
\hline Paciente & $\begin{array}{c}\text { Número } \\
\text { de stents }\end{array}$ & Localización & Estado \\
\hline 1 & 3 & ACD & $\begin{array}{l}\text { Dos permeables y } \\
\text { uno ocluido } \\
\text { (el más distal) }\end{array}$ \\
2 & 2 & ADA y ACX & Permeables \\
3 & 1 & ADA & Permeable \\
4 & 1 & ADA & Permeable \\
5 & 1 & ADA & Permeable \\
\hline
\end{tabular}

ACD: arteria coronaria derecha, ADA: arteria descendente anterior, ACX: arteria circunfleja.

Tabla III. Enfermedad ateromatosa coronaria.

\begin{tabular}{|c|c|c|c|c|c|c|}
\hline \multicolumn{3}{|c|}{ Severidad de la lesión } & \multicolumn{2}{|c|}{$\begin{array}{l}\text { Número de arterias } \\
\text { comprometidas }\end{array}$} & \multicolumn{2}{|l|}{ Arteria comprometida } \\
\hline No significativa & & $27 \%$ & Una & $43 \%$ & Arteria descendente anterior & $45 \%$ \\
\hline \multirow[t]{5}{*}{ Significativa } & & & Dos & $39 \%$ & Arteria circunfleja & $29 \%$ \\
\hline & Moderada & $37 \%$ & & & & \\
\hline & Severa & $22 \%$ & Tres & $18 \%$ & Arteria coronaria derecha & $20 \%$ \\
\hline & Obstrucción & $14 \%$ & & & & \\
\hline & & & & & Tronco coronario izquierdo & $6 \%$ \\
\hline
\end{tabular}

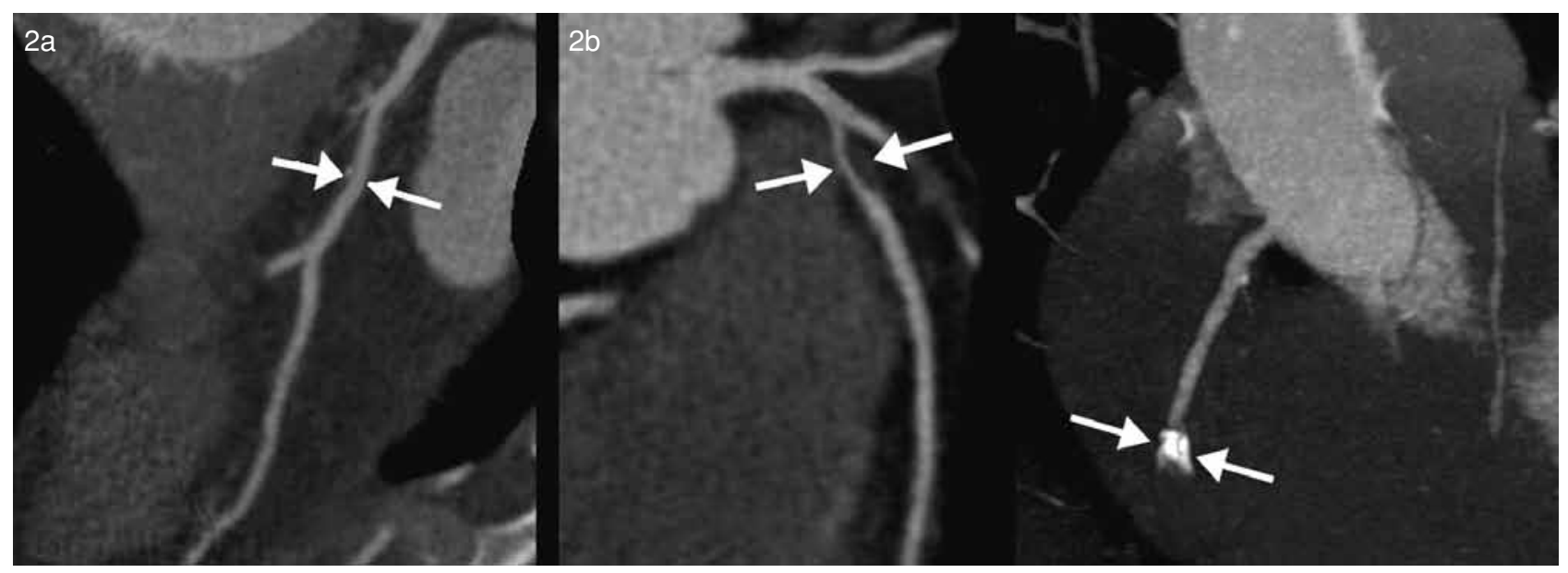

Figura 2. Enfermedad ateromatosa de arterias coronarias (flechas). a) Placa parcialmente calcificada con estenosis no significativa (<50\%) en el tercio medio de la arteria descendente anterior. b) Placa no calcificada con estenosis moderada (51-70\%) en el tercio proximal de la arteria descendente anterior. c) Placa calcificada con oclusión en el tercio medio de la arteria coronaria derecha. 


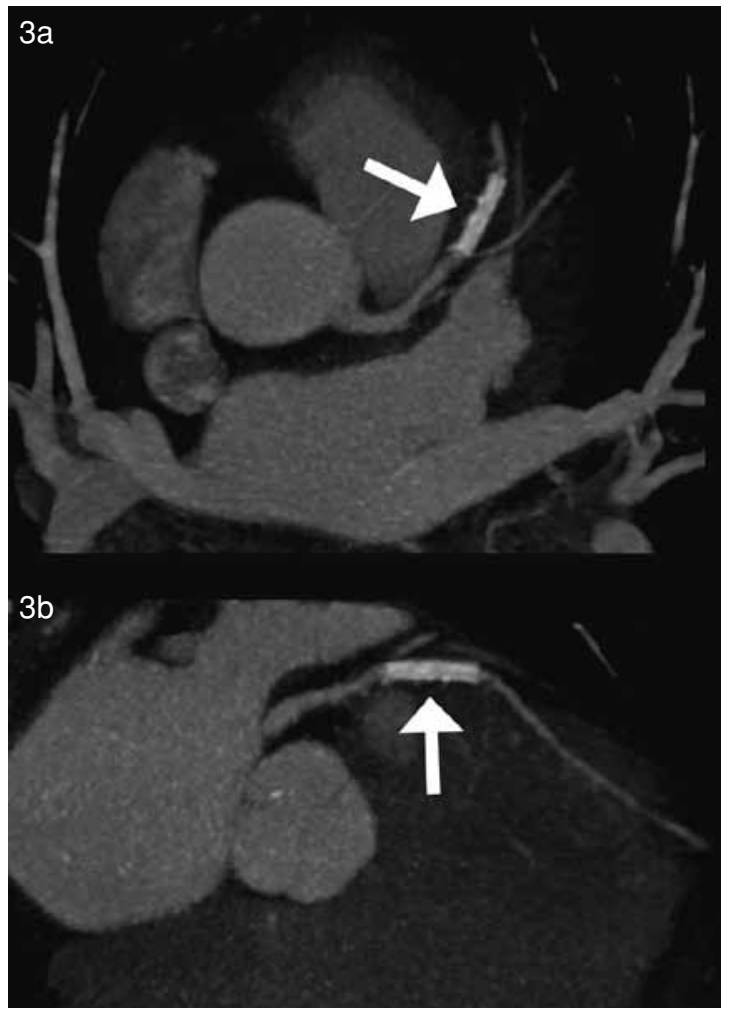

Figura 3. Stent permeable en el tercio medio de la arteria descendente anterior (flecha en imagen a y b).

Dos pacientes presentaron fístulas coronarias, una de ellas arterio-venosa (Figura 5) y la otra desde la arteria pulmonar.

Doce pacientes de la muestra tenían además coronariografía realizada en nuestro hospital. En ocho de estos pacientes se demostró una estenosis significativa con buen correlato entre los hallazgos del Angio-TC y la coronariografía (Figura 6), en dos existió sobreestimación de la severidad de la enfermedad ateromatosa coronaria en el Angio-TC y en los otros dos el Angio-TC coronario correspondió a examen de control luego de la realización de angioplastía coronaria con stent.

Catorce pacientes presentaron hallazgos cardiovasculares no coronarios. El más frecuente fue la valvulopatía aórtica, de la cual se registraron 4 casos (dos válvulas bicúspides, una válvula estenótica y una válvula con vegetaciones) (Figura 7). Le siguen en frecuencia las secuelas de infarto miocárdico, con 3 casos, todos en localización ántero-septo-apical (Figura 8). Con menor frecuencia se registró patología de la aorta torácica (dos casos, un aneurisma de aorta ascendente y una secuela de úlcera penetrante en aorta descendente) y miocardiopatías (dos casos, una miocardiopatía hipertrófica apical y una miocardiopatía no compactada). Por último, se registraron casos únicos de comunicación interauricular tipo ostium secundum, ventrículo único con cirugía de Fontan modificada (Figura 9) y derrame pericárdico.

\section{Discusión}

El Angio-TC de arterias coronarias es un examen útil para caracterizar la enfermedad ateromatosa coronaria. Meta-análisis internacionales lo han comparado con la coronariografía, demostrando alta sensibilidad y especificidad del método, en especial con el uso de tomógrafos de 64 o más canales ${ }^{(13)}$. Sin embargo, otros meta-análisis muestran que en el análisis por paciente la especificidad del método

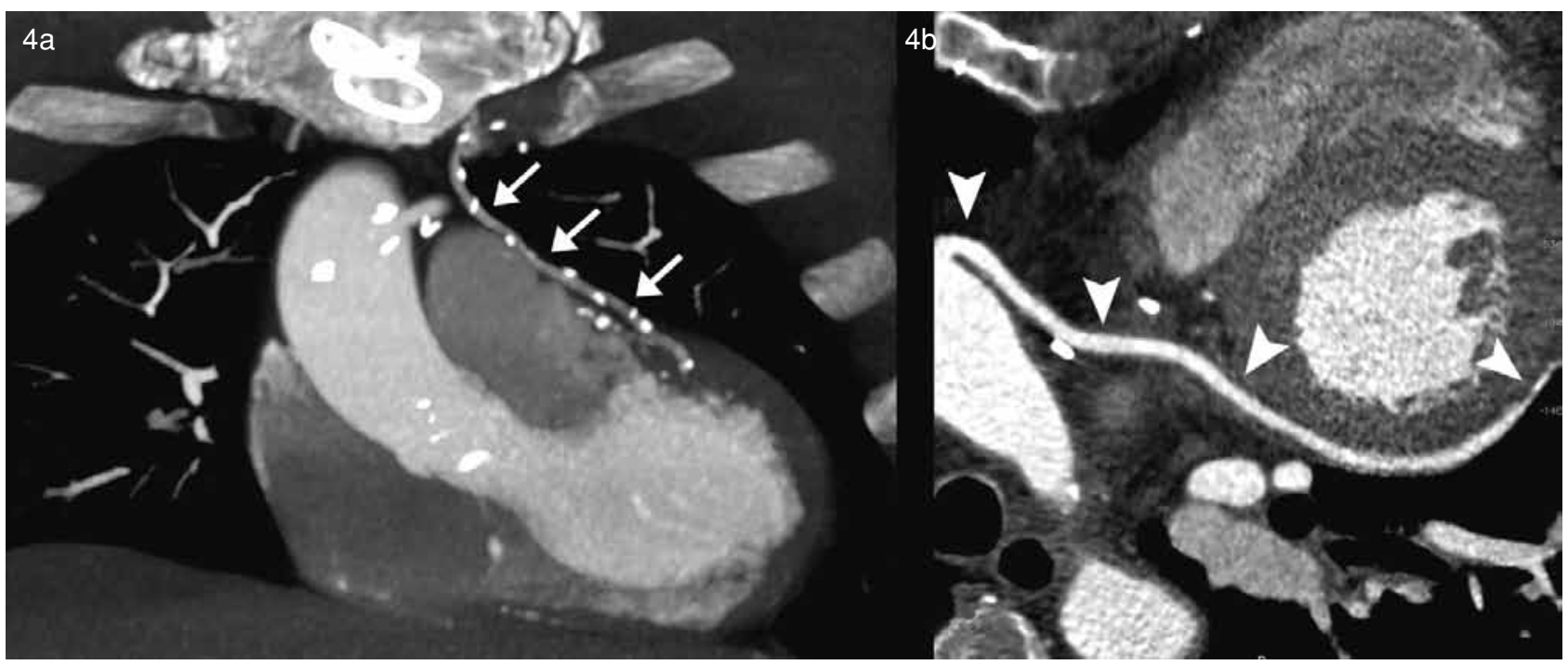

Figura 4. Bypass coronarios. a) Puente de arteria mamaria interna izquierda sobre arteria descendente anterior permeable (flechas). b) En el mismo paciente, puente venoso aorto-coronario sobre arteria marginal obtusa permeable (cabezas de flecha). 
Tabla V. Bypass coronarios

\begin{tabular}{|c|c|c|c|}
\hline Paciente & $\begin{array}{c}\text { Número de } \\
\text { bypass }\end{array}$ & \multicolumn{1}{|c|}{ Tipo } & Estado \\
\hline 1 & 3 & $\begin{array}{l}\text { (1) AMI sobre ADA } \\
\text { (2) venoso aorto-coronario sobre ADA } \\
\text { (3) venoso aorto-coronario sobre AMO }\end{array}$ & $\begin{array}{l}\text { Ocluido } \\
\text { Ocluido } \\
\text { Permeable }\end{array}$ \\
\hline 2 & 3 & $\begin{array}{l}\text { (1) AMI sobre ADA } \\
\text { (2) venoso aorto-coronario sobre ACX } \\
\text { (3) venoso aorto-coronario sobre ADP }\end{array}$ & $\begin{array}{l}\text { Ocluido } \\
\text { Permeable } \\
\text { Permeable }\end{array}$ \\
\hline 3 & 3 & $\begin{array}{l}\text { (1) AMI sobre ADA } \\
\text { (2) venoso aorto-coronario sobre ADA } \\
\text { (3) venoso aorto-coronario sobre AMO }\end{array}$ & $\begin{array}{l}\text { Permeable } \\
\text { Ocluido } \\
\text { Permeable }\end{array}$ \\
\hline
\end{tabular}

AMI: arteria mamaria interna, ADA: arteria descendente anterior, ACX: arteria circunfleja, AMO: arteria marginal obtusa, ADP: arteria descendente posterior.

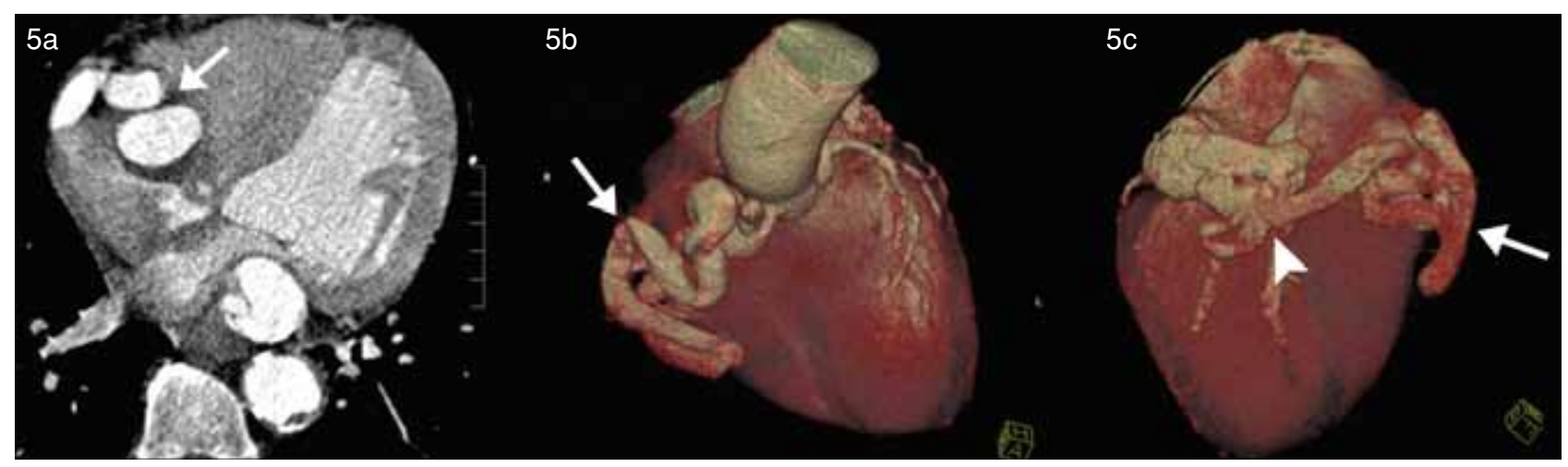

Figura 5. Fístula arterio-venosa de la arteria coronaria derecha (flechas), con drenaje a la aurícula derecha a través del seno coronario (cabeza de flecha en la imagen c). a) Corte axial. b, c) Imágenes VRT.

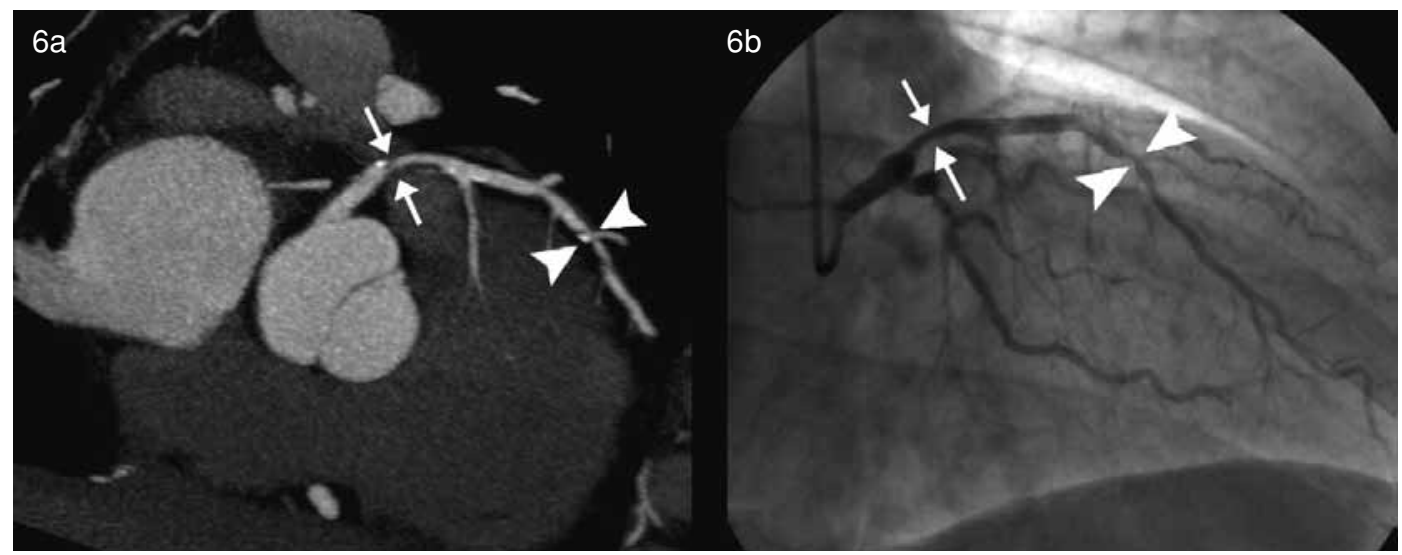

Figura 6. Correlato entre los hallazgos del Angio-TC y la coronariografía en enfermedad ateromatosa coronaria. a) Reconstrucción curva del Angio-TC que muestra una placa ateromatosa parcialmente calcificada con estenosis moderada (51-70\%) en el tercio proximal de la arteria descendente anterior (flechas) y otra placa calcificada con estenosis severa (71-99\%) en el tercio medio de la misma arteria (cabezas de flecha). c) La coronariografía realizada 14 días después confima la presencia de la placas con estenosis moderada y severa en el tercio proximal y medio de la arteria descendente anterior respectivamente (flechas y cabezas de flecha respectivamente). 


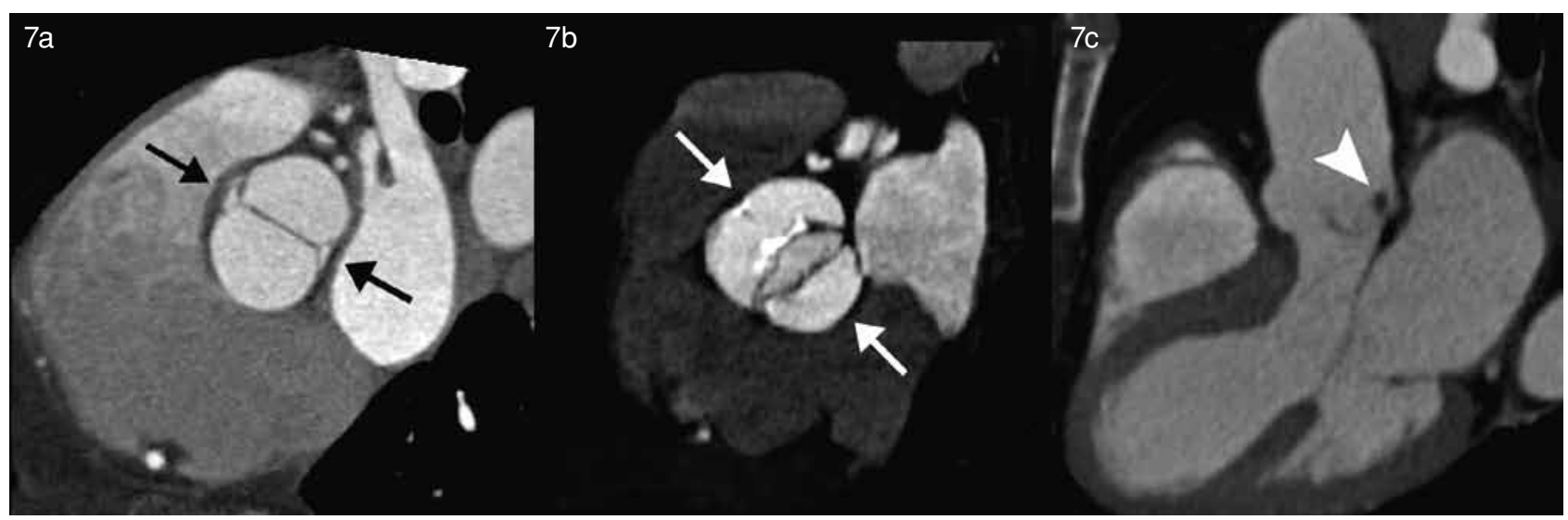

Figura 7. Valvulopatía aórtica. a) Válvula bicúspide (flechas). b) Estenosis aórtica, con calcificación y fusión parcial de velos y disminución del área valvular en imagen tomada durante la sístole (flechas). c) Vegetación dependiente del velo posterior de la válvula aórtica (cabeza de flecha) en un paciente con endocarditis infecciosa.

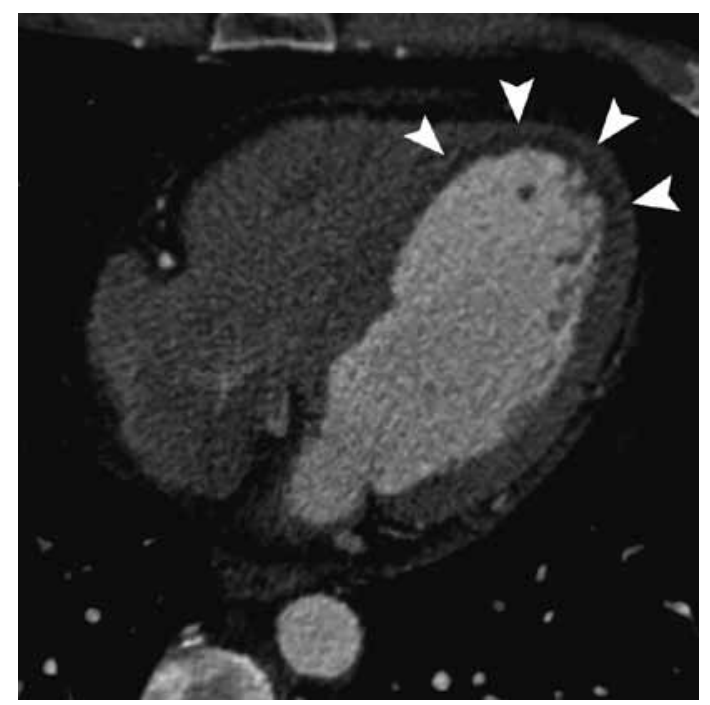

Figura 8. Secuela de infarto miocárdico ántero-septo-apical. Miocardio adelgazado con área hipodensa de remplazo fibroadiposo subendocárdica (cabezas de flecha).

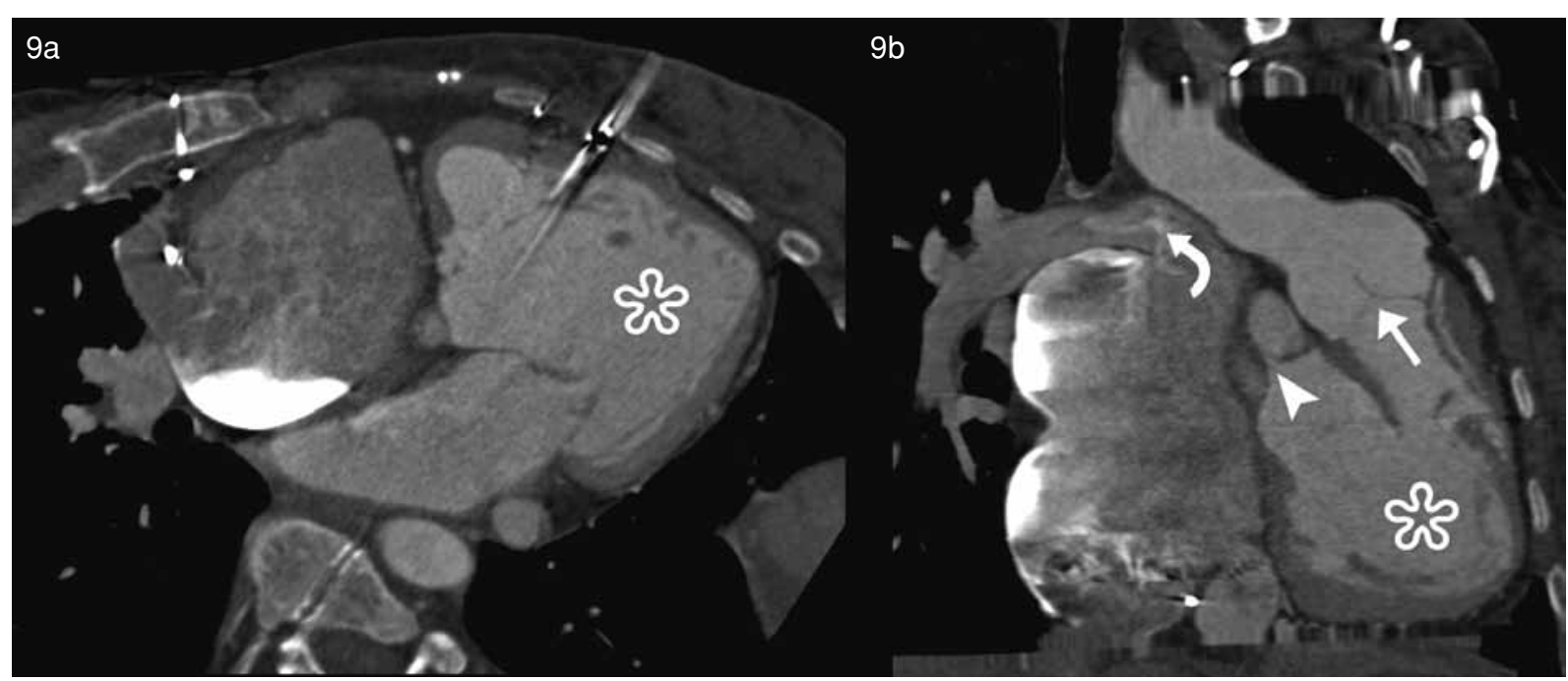

Figura 9. Ventrículo único operado con cirugía de Fontan modificada. Cavidad ventricular única (asterisco), que comunica con dos tractos de salida, uno hacia la raíz de la aorta (flecha recta en la imagen b) y otro hacia la arteria pulmonar (cabeza de flecha en la imagen b). La paciente ha sido operada, con comunicación de la aurícula derecha con la bifurcación de la arteria pulmonar (flecha curva en la imagen b). 
es relativamente baja ${ }^{(14)}$, con lo cual el número de falsos positivos se incrementa. En otras palabras, el Angio-TC tendería a sobreestimar el grado de estenosis. En nuestro estudio no fue posible calcular la sensibilidad, especificidad y valores predictivos del Angio-TC de arterias coronarias, ya que no todos los pacientes tenían coronariografía disponible con la cual comparar. Sin embargo, de los 12 pacientes que tenían coronariografía realizada en nuestro hospital, existió una buena correlación entre ambos métodos en 8 casos con estenosis significativa.

Al comparar nuestros resultados con una gran serie de coronariografías publicada en nuestro país ${ }^{(3)}$, resulta interesante ver que en términos generales el análisis de la anatomía coronaria es similar, al igual que el análisis de la enfermedad ateromatosa coronaria en cuanto a severidad de la enfermedad, número de arterias comprometidas y arterias comprometidas según frecuencia.

No existen trabajos chilenos que demuestren la utilidad del Angio-TC de arterias coronarias en el estudio de la enfermedad ateromatosa coronaria ni en el estudio de otras alteraciones coronarias menos frecuentes.

Clásicamente se ha descrito que la evaluación de stents con Angio-TC es limitada, ya que la visualización del lumen se encuentra obstaculizada por artefacto de volumen parcial y endurecimiento del haz por el material metálico del stent ${ }^{(7)}$. En la actualidad, con tomógrafos de 64 o más canales y con el uso de filtros de reconstrucción adecuados, la presencia de estos artefactos ha disminuido, pero no desaparecido. Por lo tanto, la utilidad clínica actual del Angio-TC está limitada únicamente a la detección de oclusión del stent $t^{(7)}$. En nuestro estudio, sólo un stent de los ocho evaluados se encontraba ocluido, correspondiendo al stent más distal de un paciente que tenía tres stents en la arteria coronaria derecha y triple bypass coronario.

Está demostrado en la literatura que el Angio-TC de arterias coronarias tiene un rol definido en la evaluación de bypass coronarios. Con tomógrafos de 64 o más canales el rendimiento diagnóstico para evaluar permeabilidad del injerto y visualizar la anastomosis proximal y distal es alto ${ }^{(7)}$. En nuestro estudio, 4 de los 9 bypass evaluados se encontraban ocluidos, no se registraron otras complicaciones como aneurismas del injerto o dehiscencia de las anastomosis. Esta alta tasa de oclusión es concordante con lo descrito en la serie de Ugalde et $\mathrm{al}^{(3)}$, quienes reportan que dos tercios de los bypass que evaluaron presentaban enfermedad significativa.

Las variantes anatómicas del origen de las arterias coronarias son poco frecuentes (menos del $2 \%$ de la población) ${ }^{(3,7)}$, pero pueden ser predisponente de complicaciones potencialmente mortales. El curso de las arterias coronarias anómalas es difícil de precisar con coronariografía. El Angio-TC de arterias coronarias ha demostrado ser una herramienta de gran utilidad en la evaluación de estos pacientes ${ }^{(11)}$. En nuestro estudio detectamos cinco pacientes con variantes anatómicas del origen de las arterias coronarias, de especial importancia debido a su repercusión sobre la irrigación del miocardio fueron la arteria coronaria derecha originada en el seno de Valsalva izquierdo, de curso interarterial, y la arteria coronaria izquierda originada en la arteria pulmonar.

El Angio-TC de arterias coronarias no sólo puede ser utilizado para evaluar las arterias coronarias. También presenta utilidad para la evaluación del corazón, aorta torácica y pericardio, tal como mostramos en nuestro trabajo. Un único estudio nacional descriptivo ha reportado previamente la utilidad de la técnica en la descripción de hallazgos cardíacos no coronarios ${ }^{(12)}$.

Dentro de las limitaciones de nuestro estudio se cuenta el hecho de ser retrospectivo, lo cual limita la evaluación de algunas variables (por ejemplo el motivo de solicitud del examen), tener un número relativamente pequeño de pacientes y no contar con un correlato de coronariografía en todos los pacientes. Se requiere de estudios prospectivos, con mayor número de pacientes y con correlato angiográfico para poder definir el rendimiento del Angio-TC de arterias coronarias en población chilena.

\section{Conclusión}

El Angio-TC de arterias coronarias es un examen no invasivo, útil para caracterizar la enfermedad ateromatosa coronaria y determinar la severidad de ésta. Es también útil para evaluar stents y bypass coronarios y determinar su permeabilidad, así como para describir variantes del origen de las arterias coronarias. Por último, brinda información sobre diagnósticos no coronarios a nivel cardiaco, aórtico y pericárdico.

\section{Bibliografía}

1. Ministerio de Salud, Chile. Departamento de estadísticas e información de salud. 2010.

2. Hill MN. New targeted AHA program: cardiovascular care and outcomes. Circulation 1998; 97(13): 12211222.

3. Ugalde H, Ramírez A, Dussaillant G, Ayala F, García S, Silva AM, et al. Angiografía coronaria: indicación, resultados y complicaciones en 5.000 pacientes consecutivos. Rev Med Chile 2007; 135(7): 829-838.

4. Sundaram B, Patel S, Agarwal P, Kazerooni EA. Anatomy and terminology for the interpretation and reporting of cardiac MDCT: part 1, structured report, coronary calcium screening, and coronary artery anatomy. AJR Am J Roentgenol 2009 Mar; 192(3): 574-583.

5. Sundaram B, Patel S, Agarwal P, Kazerooni EA. Anatomy and terminology for the interpretation and reporting of cardiac MDCT: part 2, CT angiography, cardiac function assessment, and noncoronary and 
extracardiac findings. AJR Am J Roentgenol 2009 Mar; 192(3): 584-598.

6. Kimura ET, Alexánderson E, Vázquez-Lamadrid J, Pale $\mathrm{R}$, Talayero JA, Cruz P, et al. Tomografía computada multidetector de arterias coronarias: estado del arte. Parte I: aspectos técnicos. Arch Cardiol Mex 2007; 77(2): 137-149.

7. Meave A, Alexánderson $E$, Rodríguez $M$, Meléndez G, Martínez A, Sierra C, et al. Tomografía computada multidetector de arterias coronarias: estado del arte. Parte II: aplicaciones clínicas. Arch Cardiol Mex 2008; 78(2): 195-209.

8. Bastarrika G, Lee YS, Huda W, Ruzsics B, Costello P, Schoepf UJ. CT of coronary artery disease. Radiology. 2009; 253(2): 317-338.

9. Mollet NR, Cademartiri F, van Mieghem CA, Runza G, McFadden EP, Baks T, et al. High-resolution spiral computed tomography coronary angiography in patients referred for diagnostic conventional coronary angiography. Circulation 2005; 112(15): 2318-2323.

10. Ropers D, Rixe J, Anders K, Küttner A, Baum U, Bautz W, et al. Usefulness of multidetector computed tomography with $64-x$ 0.6-mm collimation and $330-\mathrm{ms}$ rotation for the noninvasive detection of significant coronary artery stenoses. Am J Cardiol 2006; 97(3): 343-348.

11. Schmitt R, Froehner S, Brunn J, Wagner M, Brunner $\mathrm{H}$, Cherevatyy $\mathrm{O}$, et al. Congenital anomalies of the coronary arteries: imaging with contrast-enhanced, multidetector computed tomography. Eur Radiol 2005; 15(6): 1110-1121.

12. Alegría J, Sanhueza P, Ríos G, Soffia P, Schiappacasse $G$, Bosch E, et al. Hallazgos cardiacos no coronarios en coronariografía por tomografía computada. Rev Chil Radiol 2007; 13(2): 80-83.

13. Vanhoenacker PK, Heijenbrok-Kal MH, Van Heste R, Decramer I, Van Hoe LR, Wijns W, et al. Diagnostic performance of multidetector $\mathrm{CT}$ angiography for assessment of coronary artery disease: meta-analysis. Radiology 2007; 244(2): 419-428.

14. Hamon M, Biondi-Zoccai GG, Malagutti P, Agostoni P, Morello R, Valgimigli $M$, et al. Diagnostic performance of multislice spiral computed tomography of coronary arteries as compared with conventional invasive coronary angiography: a meta-analysis. J Am Coll Cardiol 2006; 48(9): 1896-1910.

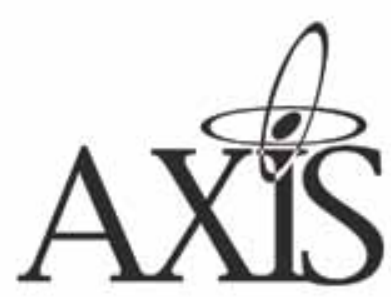

A X I S M E D I C A

Equipos de Imagen Médica

\section{VENTA DE EQUIPOS}

Radiológicos convencionales y digitales

Ultrasonidos

Mamografía covencional y digital

Densitometría osea
MONITORES PROFESIONALES Y RADIODIAGNÓSTICO

SERVICIO TÉCNICO

LABORATORIO PROPIO

CONVENIOS

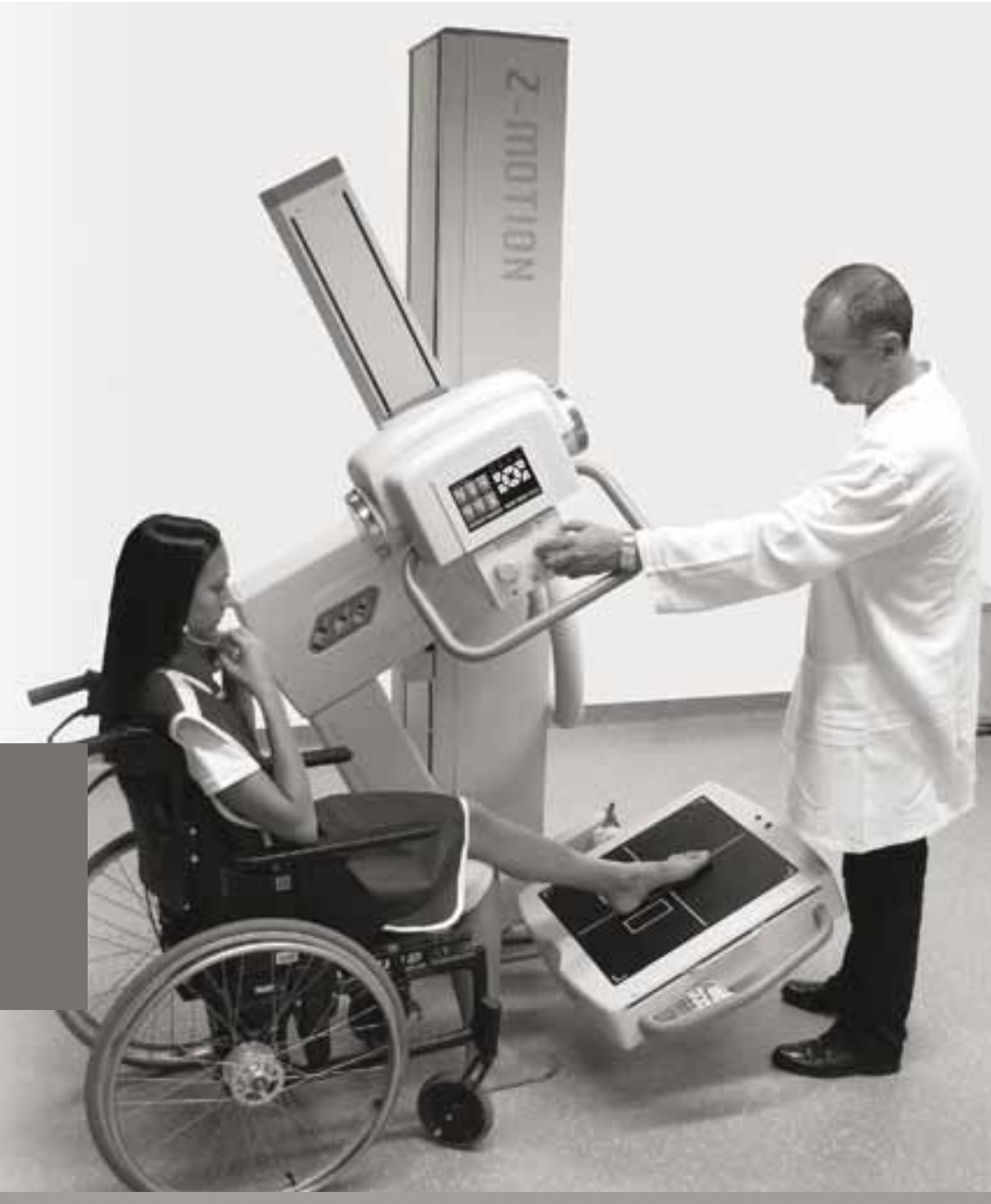

